\title{
All This Can Happen (2012) de Siobhan Davies et David Hinton
}

\author{
Nicolas Villodre, Centre National de la Danse
}

\section{Résumé}

Cet article aborde All This Can Happen du point de vue esthétique et rapporte cette œuvre née de l'étroite collaboration entre le cinéaste de danse David Hinton et la chorégraphe contemporaine Siobhan Davies à I'histoire du cinéma d'avant-garde. En apparence, All This Can Happen n'est pas un film de danse, bien que son montage rythmique fasse de l'œil aux films sturcturels des années soixante-dix et ait à voir avec la danse. Ce n'est pas un simple opus de chutes trouvées, aléatoirement agencées, puisque les archives ont été soigneusement choisies. Les plans subliminaux, la juxtaposition d'images multiples, les grattages d'émulsion, les signes graphiques, les éléments précinématographiques associés aux contrepoints sonores y produisent de fascinants effets, d'étranges hallucinations, de pures abstractions, des états de rêve éveillé, déjà là chez Georges Méliès, Abel Gance, Maya Deren et... David Hinton. Comme tout chef d'œuvre digne de ce nom, le film gagne à être revu.

Mots clés: cinéma élargi, avant-garde, écran divisé, rythme, simultanéité, hypergraphie

Ce texte vise à fournir un contexte historique permettant de considérer les aspects esthétiques et techniques du film All This Can Happen. ${ }^{1}$ II retrace le développement de diverses pratiques dans les domaines de l'image, du son et de la performance qui ont généré de nouvelles approches liées au support filmique lui-même, de même qu'aux formats et aux conditions de projection. En s'appuyant sur les films élargis et sur des expériences similaires amorcées dès l'avènement du cinématographe, cet article met en évidence un vaste corpus de mouvements artistiques et de projets qui résonnent avec le film né de la collaboration entre Siobhan Davies et David Hinton. All This... secoue le spectateur immédiatement, dès la première vision. Ce dernier pourra le revoir indéfiniment par la suite, s'il en a la possibilité, certain d'y déceler à chaque fois du nouveau, que ce soit dans le contenu - quelque plan subliminal, quelque rapprochement incongru d'images dont on cherchera en vain à connaître la source, tel ou tel signe graphique, accident pelliculaire ou effet photochimique lui ayant échappé au prime abord - ou bien dans la forme, en raison, principalement, du parti pris expérimental des auteurs. Pour créer ce film "large", dans tous les sens du terme, c'està-dire ambitieux dans son geste et son propos, polysémique, ample, littéralement, proche de l'expanded cinema - domaine qui va, disons pour donner une idée, du 
Napoléon (1927) d'Abel Gance au Chelsea Girls (1966) d'Andy Warhol -, Davies et Hinton ont recouru au "split screen" 2 et remis au goût du jour la structure du diptyque et du polyptyque jadis utilisée par les peintres. ${ }^{3}$ Leur réalisation nous permet d'aborder des questions qui ont intéressé les commentateurs, dès les années vingt, et qui restent toujours actuelles, comme celle de l'adaptation d'une œuvre littéraire - en I'occurrence, La Promenade (1917) ${ }^{4}$ de Robert Walser -, celle des interventions graphiques et de la valeur plastique de l'image - de sa cinégraphie -, celle, psychophysiologique, de la perception multi-événementielle par le spectateur. Autant de points artistiques, esthétiques et quelquefois aussi matériels, qui ont leur histoire, souvent liée à celle des avant-gardes.

\section{Pré-texte}

Prenant pour point de départ le récit de Robert Walser, les co-auteurs empruntent des chemins de traverse, s'écartent quelquefois du texte pour des raisons purement artistiques. Ils ont estimé nécessaire de condenser le récit et, pris par la logique interne du montage, ont même interverti quelque scène - on pense au passage consacré à la librairie qu'on trouve au début du livre, tout de suite après une description de couvrechefs masculins et qui est intercalé dans le film entre la brève séquence de plumes, de rubans, de chapeaux féminins et la digression sur les cafés. Leur extension du cadre de références entraîne des anachronismes puisque les images débordent un peu le lieu et la période de la promenade réelle (ou fantasmée) de Walser. Ces altérations sont directement liées à la problématique de l'adaptation littéraire par l'image. Et à la dialectique abstraction-figuration qui résulte de l'adjonction de prises de vue à du texte, quel qu'il soit. Les auteurs ne cherchent pas à substituer ou à effacer la prose de Walser par un matériau audiovisuel, qui serait plus "moderne" ou englobant. Cette quête serait par avance vouée à l'échec. Ils se bornent donc à proposer des équivalents rétiniens pouvant "rendre" l'univers, restituer les situations et les protagonistes décrits par l'écrivain suisse.

On sait que tout cela peut arriver... dès qu'on touche au livre, au verbe, au sacré. Lorsque, en sus, c'est l'art le plus profane qui s'attaque au tabou et s'attelle sans complexe à cette besogne. Rien de tel, ici : malgré l'altération, la traduction, l'interprétation de Walser par des créateurs d'une autre époque et d'un autre médium, le texte lu (en anglais et non en allemand), accourci (d'une centaine de pages, il se résume quelques "noyaux durs", si l'on peut dire, s'agissant d'un récit n'en ayant pas, dans lequel contenu et forme obéissent au principe de flânerie, de baguenauderie, de musardise), conserve son intensité évocatoire. Cela, grâce au savoir-faire de DaviesHinton, au soin apporté au mixage, aux recherches documentaires, au montage de type structuraliste ou structurel, au photogramme près, de Danny McGuire (et aussi, paradoxalement, aux gels d'image !), sans oublier la parfaite diction du comédien qui nous le restitue en voix off, John Heffernan. Ce film ne détourne ni le contenu du livre ni l'état d'esprit qui s'en dégage. En ce sens, il n'atteint pas au droit moral de l'auteur, 
par nature "perpétuel et imprescriptible" (du moins, en droit français). ${ }^{5}$ Cette question du détournement, ${ }^{6}$ méthode créatrice utilisée et justifiée par Lautréamont, proche de celle du collage, la séparation ontologique image-son ainsi que la mise en cause de l'élément photographique sont à la base du cinéma lettriste et situationniste que nous allons maintenant évoquer.

\section{Hypergraphie et Hypertexte}

En 1951, le créateur du lettrisme, ${ }^{7}$ Isidore Isou, décida d'en finir avec la synchronie entre l'audio et le visuel, une des sacro-saintes règles du cinéma sonore ou du film "parlant", à la fois pour des raisons esthétiques et économiques - il faudra attendre le début des années soixante pour qu'un matériel allégé permette aux tenants du "cinéma vérité" d'enregistrer du vrai "son direct", ${ }^{8}$ certains réalisateurs de cinéma N.R.I. (narratif-représentatif-industriel, pour reprendre l'expression de Claudine Eizykman) ${ }^{9}$ comme Fellini préférant post-synchroniser systématiquement leurs films en auditorium. Cet anti-synchronisme ou "discrépance", qui annonce celle de CageCunningham dans le domaine de la danse, donne naissance à une série de films valorisant plus le texte que l'image - exception faite des réalisations de Maurice Lemaître, hypergraphiste ${ }^{10}$ qui, à la suite de Man Ray, Len Lye et MacLaren ${ }^{11}$ apporte un soin particulier aux interventions graphiques à même l'émulsion photographique ou pelliculaire - enluminures qu'Isou nomme "ciselures". ${ }^{12}$ On observera que Davies et Hinton n'hésitent pas non plus à recourir aux biffures, aux ratures et aux griffures de la pellicule qui leur permettent d'obtenir nombre d'effets visuels.

Marc-Gilbert Guillaumin (plus tard connu comme metteur en scène de théâtre sous le nom de Marc'O), producteur du film d'Isou Traité de bave et d'éternité que programma Jean Cocteau en 1951 au festival de Cannes, édita l'année suivante lon $n^{\circ} 1,{ }^{13}$ une revue poétique contenant des textes et des manifestes d'Isou, de Poucette, de Serge Berna, de Yolande du Luart, ainsi que des scénarios de films tels que L'Anticoncept de Gil J. Wolman, Hurlements en faveur de Sade de Guy-Ernest Debord et Tambours du jugement premier de François Dufrêne..$^{14}$ L'Anticoncept, comme la première version prévue du film de Debord, ${ }^{15}$ se passait totalement d'images photographiques (Wolman alternait images circulaires noires et blanches projetées sur un ballon-sonde) au profit d'une bande-son lyrique conçue comme un collages de cris, de slogans et de phonèmes dépourvus de signification précise.

\section{Quadrature du Cercle}

Le metteur en scène français Jacques Polieri, dans son texte "Architecture et scénographie", ${ }^{16}$ observe la "rupture du cercle architectural initial (arène)", son "éclosion (théâtre grec, romain, italien)" au XVIIle siècle, avant ses transformations wagnérienne (Gesamtkunstwerk) et cinématographique (diorama). "L'idéal, dans l'absurde, pour le metteur en scène (...) est de pouvoir reconstruire chaque fois son 
théâtre." ${ }^{17}$ La structure circassienne, qui a continué à hanter Appia, Craig et tous les scénographes contemporains, de Josef Svoboda ${ }^{18}$ à l'artiste équestre Bartabas. ${ }^{19}$

Du côté d'Aubervilliers, chez Zingaro, nous avons eu l'occasion d'assister, il y a quelques années, à un mélange des genres inédit où les spectateurs occupaient la piste habituellement réservée aux jeux du cirque, assis sur des gradins fixés à un tourniquet girant lentement en sens horaire, circonvenus, captifs de manèges ininterrompus de chevaux lancés au galop en sens opposé, ces derniers entrant ou sortant de la toile de fond par intermittence, tantôt en chair et en os, tantôt sous forme d'ombres vidéo-projetées. Dans ce spectacle, ${ }^{20}$ Bartabas mêlait frénétiquement chevaux, cavaliers réels, ombres et reflets de ceux-ci, projetés, par derrière ou par devant, sur un écran à $360^{\circ}$ aux jointures et raccords invisibles. Cette pièce compliquée à mettre en place, pas rentable du tout, la jauge du théâtre étant au niveau le plus bas, réalisait l'utopie scénographique des rénovateurs du théâtre et du cinéma plus haut évoqués.

\section{Expanded Cinema}

Et c'est au cinéma "élargi" que nous avons affaire ici, autrement dit à cette tendance du 7e Art qui lui fait quitter sa base, le fait "sortir de ses gonds". Non que le film Davies et Hinton lorgne du côté du théâtre, malgré l'aspect envoûtant de sa voix off, ou du hörspiel, bien que le design sonore de Chu-Li Shewring ne soit pas une simple illustration audio, un mixage redondant relevant du montage "bruitiste" ou du film sans image, film rétréci ou minimal dont le prototype demeure le Wochenende (1930) de Walter Ruttmann, cesse d'être une reproduction pour devenir une représentation ou une expérience unique - son support même, le DCP, lui assure, au contraire, théoriquement du moins, une certaine pérennité, une stabilité, une invariabilité. Mais il s'inspire de la formule ou du "format" de la polyvision, pour reprendre ce concept cher au cinéaste Abel Gance ${ }^{21}$ ainsi qu'au musicologue, théoricien du film et producteur de "cinéphonies" Émile Vuillermoz, ${ }^{22}$ ou de la projection multi-écranique, présentant des actions, des plans, des photogrammes de diverses provenance, simultanément.

Gance conçoit des triptyques visuels à partir de 1925, et en réalise plusieurs, d'une efficacité étonnante mais très compliqués à projeter, pour son Napoléon. ${ }^{23} \mathrm{D}^{\prime}$ une part, la juxtaposition de deux ou de plusieurs bandes-images vise à "en mettre plein la vue", comme le dit en France la vox populi, autrement dit à occuper la totalité du champ visuel, à meubler l'espace (comme Satie prétendait "meubler" la durée avec ses rengaines en boucles), ${ }^{24} \mathrm{~d}^{\prime}$ 'une manière byzantine ${ }^{25}$ ou orientale. ${ }^{26}$ De l'autre, l'horror vacui n'explique pas totalement cette tendance du cinéma amorcée dès ses origines. On pense par exemple au projet de Cinéorama ${ }^{27}$ conçu par l'opérateur Lumière, Raoul Grimoin-Sanson, pour l'Exposition universelle de 1900, à la recherche d'une "vision étendue" 28 que proposera l'artiste et enseignant du Bauhaus Herbert Bayer lors de 
I'Exposition des Arts décoratifs de 1930 à Paris, ${ }^{29}$ encerclant le visiteur, sans l'immobiliser (la liberté du regard étant le but véritable de ce type d'expériences), de tirages photographiques accrochées perpendiculairement, sur différents plans (idée qu'il systématisera en 1936 en concevant le dispositif d'une "vision à 360"), au syncinéma lettriste (cf. Le Film est déjà commencé ?, 1951, de Maurice Lemaître) ${ }^{30}$ au CinemaScope $(1953)^{31}$ et aux différentes tentatives de film en 3D destinées à lutter contre la menace de la télévision et des petits écrans, au Circarama ${ }^{32}$ breveté en 1955 par Walt Disney, à la Laterna magika qu'Alfréd Radok et Josef Svoboda ${ }^{33}$ homologuèrent à l'Expo universelle de 1958 de Bruxelles.

\section{Cinéma et Illusion}

Le film élargi peut donc viser à un rendu illusionniste, comme par exemple, dans le projet de Grimoin-Sanson, une ascension en montgolfière, tantôt, au contraire, la saturation de signes produira un effet déréalisant proche de l'hallucination, du rêve éveillé, de l'abstraction. Entre ces deux pôles, tout ce qui peut accroître ou intensifier la sensation psycho-physiologique, la commotion visuelle et la fascination kaléidoscopique est permis. Dans l'esprit du Cabaret Voltaire, Picabia, qui venait d'introduire le film Entr'acte dans le déroulement du spectacle de danse Relâche, ${ }^{34}$ écrivit, pour la dernière manifestation des Ballets Suédois (et aussi de Dada), un "CinéSketch" ${ }^{\prime 35}$ dans lequel figura Marcel Duchamp en tenue d'Adam et la future femme de René Clair, Bronia Perlmutter, en tenue d'Eve. Cette courte pièce donnée au Théâtre des Champs-Elysées le soir de la Saint Sylvestre 1924 annonce, pour des historiens d'art tels que Franck Claustrat, ${ }^{36}$ le "happening", I'"event", voire la "performance" (au sens galeriste et non plus théâtral de ce terme). Quelques années plus tard, en 1928, Germaine Dulac utilisa le cinéma pour renforcer une scène de tempête dans l'opéra $L a$ Tour de feu. ${ }^{37}$ Dans les années 60, les cinéastes expérimentaux s'adonnent au film "élargi", qu'ils traitent, non plus comme un progrès artistique ou technique, mais comme un genre en soi, entre le "happening"38 (1957) de Kaprow et I'"installation" galeriste ou muséale.

En 1963, l'Américaine Barbara Rubin réalise en 24 heures Christmas on Earth, en décidant de projeter les deux bobines de 34 minutes chacune non pas à la suite l'une de l'autre, non pas côte à côte, mais l'une dans l'autre, un petit rectangle mis en abyme dans un plus grand venant perturber la vision ou, au contraire, la stimuler en la titillant. ${ }^{39}$ Deux ans plus tard, son mentor, Jonas Mekas, organise le festival "Expanded Cinema" à la Film-Makers Cinematheque qui révèle deux innovateurs dans ce domaine : Stan VanDerBeek et Robert Whitman. ${ }^{40}$ Le premier échafaude un espace de "vision intégrale", le Movie Drome ${ }^{41}$ à l'intérieur d'un silo à grains new-yorkais. Rubin présente alors le Velvet Underground à Andy Warhol, lequel ne tarde pas à se lancer dans l'expanded avec son diptyque en $16 \mathrm{~mm}$ et en son direct, Chelsea Girls (1966). Contrairement aux opus de Rubin et de Warhol, Torse (1977), film de danse à deux écrans de Merce Cunningham ${ }^{42}$ et Charles Atlas, demande une aussi grande précision 
que le Napoléon de Gance pour être projeté, les deux bobines $16 \mathrm{~mm}$ devant être synchrones à l'image près.

Simultanéisme

Certes, montage et mixage visent indiscutablement, dans le cas qui nous occupe, à une forme de synchronie, même si, par bien des aspects, celle-ci est atteinte par des effets de décalage ou de délai (delay) contrapunctiques, la bande images - l'image mérite ici d'être écrite au pluriel - annonçant assez souvent ce qui adviendra dans la colonne sonore - et inversement. L'impression de "film total" est renforcée par cette recherche d'unisson et le "All" du titre du film n'est pas qu'une promesse de Gascon, une note d'intention auteuriste, une coquetterie, mais bel et bien un programme en tous points respecté. La "polyrythmie" chère à Ricciotto Canudo, ${ }^{43}$ I'inventeur de l'expression "7e Art" qui se déroule verticalement au cours de la projection pelliculaire, suivant le défilement temporel des pistes réservées à l'audio et de la bande centrale ou part du lion occupée par l'image - et de nos jours horizontalement, de gauche à droite, sur la timeline des logiciels de montage ou de lecture numérique ! - trouve son équivalent visuel à l'intérieur du cadre, dans l'instantané ou le photogramme, quelquefois souligné par l'arrêt sur l'image.

Le cadre éclaté du film, le parti-pris de fragmentation visuelle, l'option du collage déréalisant ou surréalisant et l'agencement à la manière d'un patchwork visuel, totalement assumés par la chorégraphe et le réalisateur de films de danse produit un choc esthétique du fait même de la simultanéité de toutes sortes de stimuli. La coïncidence, au sens propre de ce terme, de formes, motifs, trames, signes en principe séparés, canalisés, discontinus peut, stricto sensu, troubler le spectateur. II ne s'agit pas ici seulement d'actions, de gestes, se déroulant en même temps, mis sur le même plan comme pour justifier un emballement, une ivresse, une excitation sans objet ou découlant d'un "dynamisme" futuriste, constructiviste, généralement associé à la ville. Il s'agit d'aller dans le sens d'une "nouvelle vision", ${ }^{4}$ pour reprendre cette expression du Bauhaus, dans celui du décentrement prôné par John Cage, principe appliqué dès 1952 à l'"événement théâtral d'un genre nouveau" monté avec Merce Cunningham, ${ }^{45}$ David Tudor, Mary Caroline Richards, Charles Olson et Robert Rauschenberg au Black Mountain College. D'après le souvenir du chorégraphe, "Rien n'était censé représenter autre chose que ce qui était donné à voir, un enchevêtrement d'événements que les spectateurs pouvaient décrypter à leur guise." 46

\section{Corps du Film}

Pour lutter contre "la rampe invisible" et la "toile de fond imaginaire" 47 du théâtre, le film a dû "éteindre l'une et déchirer l'autre", ainsi que le préconisait Émile Vuillermoz. ${ }^{48}$ Cette déchirure n'est pas que symbolique. Elle ressemble à un échiquier, à un damier, à un tableau de Piet Mondrian ou de Paul Klee. Le simultanéisme profite de la sensation de toute-puissance et du "don miraculeux d'ubiquité du cinéma". ${ }^{49}$ 
Commentant avec lyrisme un film réalisé par le metteur en scène de théâtre et théoricien du naturalisme André Antoine, Les Frères corses, tiré du roman éponyme d'Alexandre Dumas, Vuillermoz ${ }^{50}$ rappelle le "pouvoir d'évocation"51 du cinéma mais aborde aussi des questions d'ordre psycho-physiologique relatives à la réception des "mille petits clichés d'une bande (...) semblables aux cellules du cerveau humain : même rapidité foudroyante de perception, même multiplicité de miroirs à facettes qui juxtaposent sans effort les horizons les plus éloignés, supprimant les distances, abolissant l'esclavage du temps et de l'espace, embrassant dans le même temps le présent, le passé et l'avenir, reflétant simultanément tous les points cardinaux, et nous transportant en un dixième de seconde d'une extrémité à l'autre de l'univers."

Au départ, David Hinton souhaitait utiliser un corpus essentiellement précinématographique, et plus particulièrement, recourir aux bandes chronophotographiques d'Etienne-Jules Marey et de son assistant Georges Demenÿ. Selon le réalisateur, la production du film n'a pas pu obtenir le concours de la direction de la Cinémathèque Française qui avait alors la garde de ces trésors sauvegardés par son fondateur, Henri Langlois. Grâce au cinéaste scientifique Jean-Dominique Lajoux et, surtout, à l'aide du BFI, Hinton et Davies ont toutefois pu insérer quelques images de Marey et Demenÿ. Le BFI leur a donné accès à une riche matière, tous genres cinématographiques confondus, qui va du film de ville (Berlin, Die Sinfonie der Großstadt, 1927 de Ruttmann) au found footage, en passant par les films d'amateur, les actualités, des sujets pré-montés comme des défilés de mode. D'autres archives, publiques et privées, ont joué le jeu, qui ont donné accès à des extraits ou à des images fixes dont ils sont ayants droit ou conservateurs. ${ }^{52}$

Aux cinquante nuances du noir et blanc se sont ajoutées celles du film couleur, des teintes les plus froides aux plus chaleureuses. Les arrêts sur image, les éraflures de la pellicule, les regards et "poils caméra", les taches et les accidents malencontreux sont exploités plastiquement, nullement refoulés, au contraire, valorisés. En dédoublant le cadre, en démultipliant les vignettes, en étageant les plans et les prises les plus hétérogènes ou discontinus, en apposant et opposant par endroits noir et blanc et couleur, le film a inventé son propre support. Riche en contenu et plus prodigue encore en signifiants, monté non à la seconde mais au $1 / 25$ e de seconde (à l'image près), comme le Ballet mécanique (1924) également signé à deux mains (celles de Dudley Murphy et de Fernand Léger), All This... a condensé le récit poétique de Walser comme pour le faire danser. Monté certes par associations d'idées, il a surtout suivi une logique musicale. En ce sens, on peut dire qu'il a trouvé sa rythmique. 


\section{Biographie}

Nicolas Villodre a fait des études d'arts plastiques à l'Université Paris-I, couronnées en 1983 par une thèse de Ille cycle. II a participé au mouvement du cinéma expérimental, particulièrement dynamique en France à la fin des années 70. II a travaillé 30 ans à la Cinémathèque de la Danse et a intégré le CND en 2013. Parallèlement, il a publié nombre d'articles sur la danse et sur le 7e Art dans divers supports, papier et net confondus.

Email: villodre@noos.fr

\section{Notes}

${ }^{1}$ Davies \& Hinton, All This Can Happen.

2 Dans le cinéma dominant, le modèle du genre est sans doute The Thomas Crown Affair (1968) de Norman Jewison, suggéré au cinéaste par deux exemples d'écrans multiples présentés à l'Exposition universelle de Montréal, en 1967: In the Labyrinth de Roman Kroiter et Colin Low ainis que A Place to Stand de Christopher Chapman. À la Foire internationale de New York de 1964, IBM montra une installation de 17 écrans conçue par Ray et Charles Eames et un court métrage en forme de triptyque, To Be Alive, de Francis Thompson, dont s'inspira John Frankenheimer pour son Grand Prix (1966). Le split screen est plus proche du collage ou du photomontage que de la photo "composite" du XIXe siècle ou même du "vortographe" d'Alwin Langdon Coburn (1882-1966), qui tendait nettement à l'abstraction. L' "image multiple" produite par juxtaposition est également distincte de la photo "kaléidoscopique," généralement associée au rêve ou à l'hallucination dans le cinéma de fiction, obtenue au moyen de lentilles prismatiques par des cinéastes d'avant-garde tels que Dudley Murphy (1897-1968), dans Black and Tan Fantasy ou dans Le Ballet mécanique. (Cf. Delson, Dudley Murphy).

${ }^{3}$ Nous emploierons les expressions "cinéma élargi", "film large" et "expanded cinema" qui rendent comptent de l'hybridité de ces moyens techniques et englobent la polyvision d'Abel Gance évoquée infra et le "split screen" ou "écran divisé."

${ }^{4}$ Walser, Der Spaziergang. La première version anglaise est utilisée dans le film: Walser, The Walk and Other Stories.

${ }^{5}$ Cf. article L 121 du Code français de la Propriété industrielle. Loi du 92-597 1992-0701, publiée au JORF le 3 juillet 1992 : "L'auteur jouit du droit au respect de son nom, de sa qualité et de son œuvre. Ce droit est attaché à sa personne. II est perpétuel, inaliénable et imprescriptible. II est transmissible à cause de mort aux héritiers de l'auteur. L'exercice peut être conféré à un tiers en vertu de dispositions testamentaires." 
${ }^{6}$ Pour définir la notion de détournement, Debord reprend un extrait de Poésies II de Lautréamont, sans citer sa source : “Le plagiat est nécessaire. Le progrès I'implique. II serre de près la phrase d'un auteur, se sert de ses expressions, efface une idée fausse, la remplace par l'idée juste." Guy-Ernest Debord, La Société du spectacle, Paris, 1967, chapitre 8, paragraphe 207.

${ }^{7}$ Le "Lettrisme" a quelquefois pu être traduit... littéralement et non phonétiquement en anaglais par "Letterism" au lieu de "Lettrism", terme qui a fini par s'imposer.

${ }^{8}$ En France, il s'agit de la caméra $16 \mathrm{~mm}$ Eclair conçue par Coutant synchronisée par quartz au magnétophone transistorisé à of bandes $1 / 4$ de pouce Nagra mis au point par Kudelski.

${ }^{9}$ Eizykman, La Jouissance-Cinéma, 10.

${ }^{10}$ L'hypergraphie ou la métagraphie proposé par les lettristes en 1950 est un système qui prône l'usage de toutes sortes de signes possibles et imaginaires (lettres, sons, gestes, etc.). Isidore Isou observe qu'en peinture un élément figuratif placé dans une composition abstraite suffit à la changer en tableau figuratif tandis qu'un seul signe introduit dans un tableau, figuratif ou non, le transforme en création hypergraphique, le signe l'emportant sur la matière référentielle (Isou, Le Lettrisme et l'Hypergraphie).

${ }^{11}$ Cf. Brenez \& Lebrat, Jeune, Dure et Pure; Man Ray \& Tzara, Tristan Les Champs Délicieux; Bouhours, \& Horrocks, Len Lye; Bassan,. Norman McLaren; Holter, Photography Without a Camera; Villodre, "Les Récréations Photographiques à la Fin du XIXe Siècle;" Villodre, "L'Anti-Photographie."

${ }^{12}$ Isou, "Esthétique du Cinéma."

${ }^{13}$ Ibid. Numéro unique d'une revue éditée par le Centre de Création, rééditée en 1999 par Jean-Paul Rocher.

${ }^{14}$ Poet, visual artist, and poster designer who later became part of the New Realism movement.

${ }^{15}$ Cf. Debord Guy. CEuvres Cinématographiques Complètes. Paris: Champ Libre, 1978 ; new publication: Gallimard, 1994. Before founding L'Internationale Situationniste, Debord, Wolman, Brau, Berna and a few others created L'Internationale Lettriste (19521957).

${ }^{16}$ Polieri, Scénographie, Sémiographie, 29.

${ }^{17}$ Ibid.

${ }^{18}$ Nous découvrîmes en 1984 au théâtre du Rond-Point un spectacle de sa fameuse Laterna magika intitulé Le Cirque enchanteur qui faisait usage de la projection de film $35 \mathrm{~mm}$ sur un écran en arc de cercle d'où donnaient l'impression de sortir clowns et acrobates de chair et d'os.

${ }^{19}$ Créateur de cabarets équestres mêlant théâtre, danse, musique extra-européenne, poésie. Bartabas a installé en 1984 sa troupe au Fort d'Aubervilliers dans un cirque en dur conçu par lui et l'architecte Patrick Bouchain, le théâtre Zingaro. Fondateur, en 2003, de l'Académie équestre de Versailles, qu'il considère comme un "corps de ballet" classique où les apprentis cavaliers pratiquent toutefois le kyudo (tir à l'arc japonais), la comédie et la danse. 
${ }^{20}$ Intitulée Darshan (2009), cette pièce offre une scénographie complexe où le public est placé au centre de l'arène tandis que chevaux et cavaliers s'animent autour de lui, passent tantôt devant, tantôt derrière, en ombres chinoises, une bande de gigantesque zootrope, le prennent d'assaut. (Cf. Solis, "Darshan, Bartabas à Pas Comptés.")

${ }^{21}$ Une partie seulement des triptyques du Napoléon a été utilisée lors des rares présentations du film depuis son avant-première en 1927 à l'Opéra de Paris. D'après Jean-Jacques Meusy (1895, n³1, Paris, 2000, p. 153-211), Gance recourait déjà au "fractionnement de l'écran" dans sa première version de J'accuse (1919). Selon Kevin Bronlow, Émile Vuillermoz proposa le terme de "polyvision" à cette formule multiécranique. (Cf. Bronlow, The Parade's Gone By, p. 559).

${ }^{22}$ Le regretté Noureddine Ghali admirait cette personnalité hors du commun. Pour lui, "Vuillermoz est une figure particulièrement méconnue de la critique et de la théorie cinématographiques françaises. Pendant la première guerre mondiale et durant les années vingt, il écrivit de très nombreux articles marqués du sceau de la lucidité et de la profondeur" (Ghali, L'Avant-garde Cinématographique, 327). Vuillermoz, qui publia une somme sur la musique, Critique musicale (1902-1960), Fayard, rééd. 2013, a fait l'objet de l'ouvrage monographique de Pascal Manuel Heu, Le Temps du cinéma : Emile Vuillermoz, père de la critique cinématographique (1910-1930).

${ }^{23}$ Gance, Napoleon.

${ }^{24}$ John Cage publia en 1949 dans la revue française Contrepoints une partition inédite d'Erik Satie 1866-1925), un modèle de musique répétitive ayant pour titre Vexations, composée selon les spécialistes en 1893, à base d'un thème et de deux variations sur un tempo "très lent" devant être repris... 840 fois de suite, ce qui en fait une pièce d'une durée d'environ 18 heures! Ce morceau fut joué au piano en 1963 à New York dans un marathon où se succédèrent John Cale, John Cage, David Tudor, Christian Wolff, Philip Corner, Viola Farber, Robert Wood, MacRae Cook, David Del Tredici, James Tenney, Howard Klein.

${ }^{25}$ Satie composa des morceaux néo-byzantins comme les Gymnopédies (1888) et, surtout, les Gnossiennes (1891) aux ornements grecs ou orientaux. Proche alors du prosateur décadent Joséphin Péladan, grande figure rosicrucienne, il écrivit pour lui L'Hymne pour le salut au drapeau du Prince de Byzance, qu'enregistra le ténor Nicolaï Gedda en 1987, qu'EMI sortit en CD en 1996. On sait aussi que les gamelans javanais invités à l'Exposition universelle de 1889 produisirent sur lui comme sur Debussy forte impression et l'incitèrent sans doute à user de la répétitivité (cf. supra Note 24 : Vexations, 1893 ainsi que la musique du film Entr'acte, 1924).

${ }^{26}$ Lorsque nous analysons le rapport des surfaces entre elles, nous nous apercevons qu'à partir d'une distance, trop lointaine ou trop proche, et, pour le film, d'une relative accélération, on ne peut plus distinguer les détails et séparer les "atomes plastiques". Cette observation découle de la "logique des densités" chère à Alexandre Papadopoulo, qui a étudié l'organisation de l'espace spiralée dans la miniature persane, l'art non figuratif de la calligraphie, l'art abstrait de l'arabesque et l'esthétique de l'horreur du vide dans L'Islam et l'art musulman. 
${ }^{27}$ La Nature, 119-122 donne une description de ce système nécessitant une dizaine de caméras synchrones et autant d'appareils de projection. Louis Lumière lui-même eut en 1899 l'idée, qu'il breveta le 29 décembre 1900, du Photorama, un système de reproduction complète de l'horizon, panorama non plus optique, comme celui de la camera obscura, mais photographique, qu'on a pu voir au Grand Palais dans le cadre de l'exposition "Lumière ! Le Cinéma inventé," 27 mars-14 juin 2015. Cf. Les Lumière font leur cinéma, 30.

${ }^{28}$ Meusy, "L'Énigme du Cinéorama," 1-16.

${ }^{29}$ Cf. Quoi, "Herbert Bayer et la vision étendue."

${ }^{30}$ Le découpage du film et les diverses interventions prévues dans la salle se trouvent dans : Lemaître, Le Film est déjà commencé ?

${ }^{31}$ Technique de l'image anamorphosée développée par la 20th Century Fox et utilisée par l'industrie hollywoodienne, de 1953 aux années soixante, basé sur l'Hypergonar et l'Anamorphoscope brevetés en 1926 par le Français Henri Chrétien. https://en.wikipedia.org/wiki/Anamorphosis

${ }^{32}$ Le premier film en Circarama, A Tour Of The West, fut projeté lors de l'inauguration de Disneyland, en juillet 1955. (Cf. Nielsen, Projecting America).

${ }^{33}$ Ibid. La Laterna Magika fut présentée à l'Exposition universelle de Bruxelles en 1958. Cf. aussi note 18.

${ }^{34} \mathrm{Cf}$. le catalogue de l'exposition à l'Opéra de Paris: Auclair, Les Ballets Suédois.

${ }^{35}$ Cette "revue," écrite tantôt "ciné-sketch," tantôt "cinescketch," conçue par Picabia pour la "soirée de gala de Rolf de Maré," ainsi annoncée par le programme, tut mise en scène et éclairée par René Clair, qui usa d'effets stroboscopiques. Man Ray, l'auteur d'une belle photo sans doute prise pendant les répétitions, y aurait tenu un rôle de bavard, tandis que Jean Börlin y aurait interprété un flic; la danseuse isadorienne Élisabeth Toulemont (alias Caryathis) s'y serait produite sur des musiques du groupe des Six ; des poèmes auraient été récités par Yvonne George ; du jazz "hot" aurait été joué par l'orchestre des Georgians. Cf. Lynn Garafola, Legacies of Twentieth-Century Dance, Middletown, Wesleyan University Press, 2005, p. 114. Selon Carole Boulbès, des équivalents théâtraux au ralenti, à l'accéléré et la recherche de simultanéité cinématographiques y auraient été proposés (Boulbès, Francis Picabia, Écrits critiques, 555).

${ }^{36} \mathrm{Cf}$. Auclair, Les Ballets Suédois.

${ }^{37}$ Drame lyrique en trois actes de Sylvio Lazzari mis en scène à l'Opéra de Paris par Pierre Chéreau, chorégraphié par Nicola Guerra, incluant une projection de mer déchaînée traversant les diverses toiles peintes du décor de Maxime Dethomas. Cette interaction film/spectacle, on la retrouve dans des ballets récents tels que Dance (1979) de Lucinda Childs, suite gestuelle synchronisée à un film $35 \mathrm{~mm}$ NB de Sol LeWitt projeté en fond de scène, ou Biped (1999), chorégraphie multimédia de Merce Cunningham ponctuée par les images géantes en 3D de lignes de force et de traces des danseurs traitées par les artistes-infographistes Shelley Eshkar et Paul Kaiser. Cf. la notice de la Bibliothèque de France FRBNF42047774 se référant à Wolff, Stéphane. L'Opéra au Palais Garnier (1875-1962). Paris: 1962. Pour Hilds : Boisseau, "La 'Dance' 
Perpétuelle de Lucinda Childs;" pour Cunningham : Dahan \& Gauville "Danse: Entretien Musical Autour."

38 Le mot "Happening" fut employé pour la première fois par Allan Kaprow en 1957 pour qualifier une "performance" artistique (Kaprow, Allan. "Happenings in the New York Scene." Art News, May, 1961, rééd. : Kaprow, Essays on the Blurring of Art and Life.

${ }^{39}$ Dans nombre d'exemples, l'effet s'obtient à la projection, la pellicule n'étant pas nécessairement plus "large" et la question de l'anamorphose ne jouant pas. C'est le cas de Man Ray qui aurait projeté des films en couleur de Méliès sur les danseurs, tous vêtus de blanc, lors d'une fête en plein air donnée en 1930 par Anna-Letizia PecciBlunt ou de Werner Nekes dirigeant les images clignotantes de son Schnitte für $A B A B A$ (1967) sur une forêt de sapins. Cf. Dominique Noguez, Cinéma \&, 117.

${ }^{40}$ Whitman présenta Prune Flat, interprété par Mimi Stark, Simone Forti et Lucinda Childs. Cf. Uroskie, Expanded Cinema, 133

${ }^{41}$ Bouhours, Quel cinéma, 166-167.

42 Pour la pièce de Cunningham, Variations V (1965), VanDerBeek utilisa plusieurs écrans sur lesquels étaient projetés des montages en $16 \mathrm{~mm}$ d'images publicitaires, de films d'animation et de série $B$ diffusés par la télévision ainsi que des déformations électroniques abstraites signées Nam June Paik. On peut voir ces films expérimentaux dans la captation du ballet réalisée l'année suivante par Arne Arnborn pour la chaîne de télévision allemande NDR.

${ }^{43}$ Guido, L'Age du rythme, 194-200.

${ }^{44}$ Moholy-Nagy, Laszlo. The New Vision and Abstract of an Artist. New York: Wittenborn, 1947.

${ }^{45}$ Cf. David Vaughan, Merce Cunningham: Fifty Years, 65.

${ }^{46}$ Ibid.

${ }^{47}$ Vuillermoz, "Devant l'Écran," 3

${ }^{48}$ Ibid.

49 Ibid.

50 Ibid.

${ }^{51}$ Ibid.

${ }^{52}$ Le Yorkshire Film Archive, British Pathé, la Wellcome Library, la London A P Archive, la British Movietone, la Religious Society of Friends in Britain, le National Grid PLC, Catalyst Housing, Les Films du Jeudi, Argos Films, Le Collège de France, le Museum of London, la Library of Congress, John Martin, la Photographic Society, le Science Museum, etc.

\section{Références}

All This Can Happen. Dir. Siobhan Davies et David Hinton. UK, 2012. Digital Video. 
A Place to Stand. Dir. Chirstopher Chapman. Canada: 1967.65 mm Film.

Auclair, Mathias (ed.). Les Ballets Suédois, Une Compagnie d'Avant-garde (1920-1925). Montreuil : Gourcuff Gradenigo, 2014.

Bassan, Raphaël. Norman McLaren, le Silence de Prométhée, Paris: Paris Expérimental, 2004.

Berlin, Die Sinfonie der Großstadt/Berlin: Symphony of a Great City. Dir. Walter Ruttman. Germany: 1927. 35 mm Film.

Black and Tan Fantasy. Dir. Dudley Murphy. USA: 1929. 35 mm Film.

Boisseau, Rosita. "La 'Dance' Perpétuelle de Lucinda Childs," Le Monde, 22 octobre 2014.

Bouhours, Jean-Michel. Quel cinéma. Paris: JRP-Ringier \& Les Presses du Réel, 2010.

Bouhours, Jean Michel et Roger Horrocks. Len Lye, Paris: Centre Pompidou, 2000.

Boulbès, Carole. Francis Picabia, Écrits Critiques. Paris: Mémoire du Livre, 2005.

Brenez, Nicole et Christian Lebrat. Jeune, Dure et Pure : une Histoire du Cinéma d'Avantgarde et Expérimental. Paris: Cinémathèque Française \& Mazzotta, 2000.

Bronlow, Kevin. The Parade's Gone By. Berkeley: University of California Press, 1968.

Cage, John. "Raison d'être de la Musique Moderne." Contrepoints, Une revue de musique, $\mathrm{n}^{\circ}$ 6. Paris: Richard Masse, 1949.

Chelsea Girls. Dir. Paul Morrissey and Andy Warhol. USA: 1966. 2 x 16 mm Film.

Christmas on Earth. Dir. Barbara Rubin. USA: 1963. 2 x 16 mm Film.

Code de la Propriété Intellectuelle, article L 121, loi 92-597 1992-07-01, JORF, 3 juillet 1992.

Dahan, Eric et Hervé Gauville. "Danse: Entretien Musical Autour de 'Biped,' Dernière Création de Merce Cunningham - Gavin Bryars en Cunningham Majeur," Libération. 11 novembre 1999.

Debord, Guy. La Société du Spectacle. Paris: Buchet/Chastel, 1967.

Delson, Susan B. Dudley Murphy: Hollywood Wild Card. Minneapolis: University of Minnesota Press, 2006.

Dufrêne, François. Tambours du Jugement Premier. Film sans image, France: 1952, rééd. 1981. 
Eizykman, Claudine. La Jouissance-cinéma. Paris: UGE, 1975.

Entr'acte. Dir. René Clair and Francis Picabia. France: 1924. 35 mm Film.

Garafola, Lynn. Legacies of Twentieth-Century Dance. Middletown: Wesleyan University Press, 2005.

Ghali, Noureddine. L'Avant-garde Cinématographique en France dans les Années Vingt. Paris: Paris Expérimental, 1995.

Grand Prix. Dir. John Frankenheimer. USA: 1966. 65 mm Film.

Guido, Laurent. L'Age du Rythme. Lausanne: Payot, 2007.

Guillaumin, Marc-Gilbert (Marc'O). Ion, 1. Paris: Centre de Création, 1952.

Heu, Pascal Manuel. Le Temps du Cinéma: Émile Vuillermoz Père de la Critique Cinématographique (1910-1930). Paris: L'Harmattan, 2003.

Holter, Patra. Photography Without a Camera. New York/Londres: VNR/Studio Vista, 1972.

Hurlements en Faveur de Sade / Howlings in Favor of Sade. Dir. Guy Debord. France: 1952. $35 \mathrm{~mm}$ Film.

In the Labyrinth. Dir. Roman Kroitor, Colin Low, and Hugh O'Connor. Canada: 1967.70 $\mathrm{mm}$ Film.

Isou, Isidore. "Esthétique du Cinéma," in Ion, 1. Paris: Centre de Création, 1952 . Fuvres de Spectacle. Paris: Gallimard, 1964. . Le Lettrisme et l'Hypergraphie dans la peinture et la sculpture contemporaines. Paris : Jean Grassin, 1961.

J'accuse. Dir. Abel Gance. France: 1919. 35 mm Film.

Kaprow, Allan. Essays on the Blurring of Art and Life. Berkeley: University of California Press, 1993.

L'Anticoncept. Dir. Gil Wolman. France: 1952. 35 mm Film.

Le Ballet Mécanique. Dir. Fernand Léger and Dudley Murphy. France: 1924. 35 mm Film. Le Film est Déjà Commencé? Dir. Maurice Lemaître. France: 1951. 35 mm Film. Les Frères Corses/The Corsican Brothers. Dir. André Antoine. France: 1917. 35 mm Film. 
Lemaître, Maurice. Le Film est Déjà Commencé? Paris: André Bonne, 1952.

Mareschal, G. La Nature, Paris: 21 juillet 1900, p.119-122.

Meusy, Jean-Jacques. "L'Énigme du Cinéorama," Archives, 37. Toulouse: Institut Jean Vigo, 1991.

Meusy, Jean-Jacques. “La Polyvision, Espoir Oublé d'un Cinéma Nouveau," 1895, 31. Paris: AFRHC, 2000.

Moholy-Nagy, Laszlo. The New Vision and Abstract of an Artist. New York: Wittenborn, 1947.

Napoléon. Dir. Abel Gance. France: 1927. 35 mm \& 3 x 35 mm Film. Nielsen, Sarah Dawn. Projecting America: Films at the Brussels World's Fair of 1958. Los Angeles: University of Southern California, 2000.

Noguez, Dominique. Cinéma \&. Paris: Paris Expérimental, 2010.

Odicino, Guillemette. "Les Lumière font Leur Cinéma." Télérama, édition spéciale, Paris: mars 2015.

Papadopoulo, Alexandre. L'Islam et l'art Musulman. Paris: Citadelles \& Mazenod, 1976.

Polieri, Jacques. Scénographie, Sémiographie. Paris: Denoël/Gonthier, 1971.

Quoi, Alexandre. "Herbert Bayer et la Vision Étendue." Vues d'en Haut, Metz: éditions du Centre Pompidou-Metz, 2013.

Ray, Man \& Tristan Tzara (preface) Les Champs Délicieux. Paris: 1922.

Satie, Erik. Mélodies. L.P. Record. Issy-les-Moulineaux: EMI Music France, 1987.

Solis, René. “Darshan, Bartabas à pas comptés.” Libération, Paris: 10 décembre 2009.

The Thomas Crown Affair. Dir. Norman Jewison. USA: 1968. 35 mm Film.

To Be Alive. Dir. Alexander Hammid and Francis Thompson. USA: 1964. 3 x 35 mm Film.

Torse. Dir. Charles Atlas and Merce Cunningham. USA: 1977. 2 x 16 mm Film

Traité de Bave et d'Éternité / Venom and Eternity. Dir. Isidore Isou. France: 1951. 35 mm Film.

Uroskie, Andrew V. Expanded Cinema and Postwar Art. Chicago: The University of Chicago Press, 2014.

Variations V. Dir. Arne Arnborn. Germany: 1966. 16 mm Film. 
Vaughan, David. Merce Cunningham: Fifty Years. New York: Aperture, 1997. Villodre, Nicolas. "Christian Schad Période Dada." Photographies, 7. Paris : mai 1985. . "Les Récréations Photographiques à la fin du XIXe Siècle." Photographies, 8, Paris : septembre 1985. . "L'Anti-Photographie." Revue d'Esthétique, 7, Paris: Privat, 1984.

Vuillermoz, Émile. “Devant l'Écran”, Le Temps, 16 avril 1917-15 mai 1918.

Vuillermoz Émile. Critique Musicale (1902-1960). Paris: L'Harmattan, 2013. Walser, Robert, Der Spaziergang. Frauenfeld \& Leipzig: Huber \& Co, 1917. Walser, Robert, The Walk and Other Stories. Trad. C. Middleton. Londres: John Calder, 1957.

Wochenende/Week-end. Dir. Walter Ruttman. Germany: 1930. 35 mm Film.

Wolff, Stéphane. L'Opéra au Palais Garnier (1875-1962). Paris: L'Entr'acte, 1962. 